Research Article

\title{
Identification of Potential Core Genes in Parkinson's Disease Using Bioinformatics Analysis
}

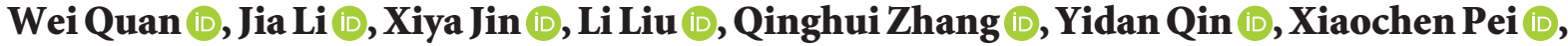 \\ and Jiajun Chen $\mathbb{D}$
}

\begin{abstract}
Department of Neurology, China-Japan Union Hospital of Jilin University, No. 126, Xian Tai Road, Changchun, Jilin 130000, China
\end{abstract}

Correspondence should be addressed to Jiajun Chen; cjj@jlu.edu.cn

Received 8 June 2021; Revised 21 August 2021; Accepted 8 September 2021; Published 18 September 2021

Academic Editor: Carlo Colosimo

Copyright (c) 2021 Wei Quan et al. This is an open access article distributed under the Creative Commons Attribution License, which permits unrestricted use, distribution, and reproduction in any medium, provided the original work is properly cited.

Purpose. This study aimed to explore new core genes related to the occurrence of Parkinson's disease (PD) and core genes that can lead to the progression of PD. Methods. The expression profile data of GSE42966, which contained six substantia nigra tissues isolated from normal individuals and nine substantia nigra tissues isolated from patients with PD, were obtained from Gene Expression Omnibus. Differentially expressed genes (DEGs) were identified, followed by functional enrichment analysis and protein-protein interaction (PPI) network construction. We then identified 10 hub genes and analyzed their expression in different Braak stages. Results. A total of 773 DEGs were identified that were significantly enriched in metabolic pathways. Ten hub genes were identified through the PPI network, namely, GNG3, MAPK1, FPR1, ATP5B, GNG2, PRKACA, HRAS, HSPA8, PSAP, and GABBR2. The expression of HRAS was different in patients with PD with Braak stages 3 and 4 . Conclusion. These 10 hub genes and the metabolic pathways they are enriched in may be involved in the pathogenesis of PD. HRAS may have potential value in predicting the progression of $\mathrm{PD}$.

\section{Introduction}

Parkinson's disease (PD) is a severe neurological disease resulting from the progressive degeneration of dopaminergic neurons located in the substantia nigra [1]. As the second most common neurodegenerative disease, PD affects approximately seven million people worldwide [2]. Currently, this disease has no cure. As the disease progresses, the patient's motor and nonmotor symptoms gradually aggravate, which seriously affects the quality of life in elderly patients.

Current research suggests that PD is caused by a combination of genetic and nongenetic factors [3]. Although researchers have confirmed that many genes are related to the occurrence of PD, including LRRK2 [4] and VPS35 [5], the specific pathogenesis is still unclear. There is a lack of understanding of the key genes that can identify the progression of PD. Therefore, it is important to identify new genes potentially involved in the manifestation and progression of PD. These genes could serve as possible new targets for the treatment of PD.

Our research aims to discover new key genes related to the occurrence of PD and core genes involved in the progression of PD. To identify differentially expressed genes (DEGs) between the substantia nigra of patients with PD and healthy individuals, bioinformatics methods were used to analyze gene expression profiling data downloaded from the Gene Expression Omnibus (GEO) database. Gene Ontology (GO) functional annotation analysis and Kyoto Encyclopedia of Genes and Genomes (KEGG) pathway enrichment analyses were performed for the DEGs identified. We then established a protein-protein interaction (PPI) network to identify hub genes related to PD. Finally, we analyzed the expression of the hub genes in patients with PD with Braak stages 3 and 4 . Braak staging is based on a hypothesis on the cause of sporadic PD proposed by Braak in 2003, which states that an unknown pathogen in the intestine may be the cause of PD. Based on the specific mode of $\alpha$-synuclein 
delivery, they proposed a PD-related pathological staging system, termed as Braak staging. Braak staging is divided into six stages according to the degree of brain involvement in patients with PD. The gradual expansion of the disease from stage 1 to stage 6 also indicates the progression of the disease $[6,7]$. Therefore, by analyzing the brain tissues of patients with PD with different Braak stages, we found that the hub genes have different expression levels in different stages; thus, examining the expression of the hub genes could help predict disease progression in PD. Through the above methods, we found that DEGs were significantly enriched in metabolic pathways and identified 10 hub genes that may be related to the pathogenesis of PD, namely, GNG3, MAPK1, FPR1, ATP5B, GNG2, PRKACA, HRAS, HSPA8, PSAP, and GABBR2. Among these, HRAS may have potential value in predicting the progression of $\mathrm{PD}$.

\section{Materials and Methods}

2.1. Data Source. In this study, gene expression datasets were obtained from the GEO database (https://www.ncbi.nlm. nih.gov/geo/). A total of 109 human PD series cases were retrieved from the database. We chose human brain substantia nigra tissue specimens for analyses as they better reflect the true differences in gene expression in patients with PD. After careful review, we choose the series GSE42966 with clinical information on Braak staging. GSE42966 was based on the Agilent GPL4133 platform (Agilent-014850 Whole Human Genome Microarray 4x44K G4112F). Data were freely available online, and our study did not involve any experiments with humans or animals performed by any of the authors.

2.2. Data Processing of DEGs. The GEO2R online analysis tool (https://www.ncbi.nlm.nih.gov/geo/geo2r/) was used to detect DEGs between PD and normal samples, and the $P$ value and $|\log \mathrm{FC}|$ were calculated. Genes that met the cutoff criteria, $P<0.05$ and $|\log \mathrm{FC}| \geq 1.0$, were considered as DEGs. Genes with $P<0.05$ and $\log \mathrm{FC} \geq 1.0$ were considered as upregulated, and genes with $P<0.05$ and $\log \mathrm{FC} \leq-1.0$ were considered as downregulated genes.

2.3. GO and KEGG Pathway Analysis of DEGs. GO analysis [8] is a commonly used method for large-scale functional enrichment research, using which gene functions can be classified into biological process (BP), molecular function (MF), and cellular component (CC). KEGG is a widely used database that stores a large amount of data on genomes, biological pathways, diseases, chemical substances, and drugs. GO annotation and KEGG pathway enrichment analyses of DEGs in this study were performed using the Database for Annotation, Visualization, and Integrated Discovery (DAVID) tool (https://david.ncifcrf.gov/) [9]. In GO annotation analysis of DEGs, $P<0.05$ and gene counts $\geq 20$ were considered statistically significant; in the KEGG pathway enrichment analysis of DEGs, $P<0.05$ and gene counts $\geq 10$ were considered statistically significant.
2.4. PPI Network Construction and Hub Gene Identification. We used the Search Tool for the Retrieval of Interacting Genes (STRING) database (http://string-db.org/) to analyze PPI information $[10,11]$. To evaluate the potential PPI relationship, the DEGs identified previously were mapped to the STRING database. PPI pairs were extracted with a combined score of $>0.7$. Subsequently, the PPI network was visualized using Cytoscape software (https://www.cytoscape. org/) [12]. Nodes with a higher degree of connectivity tend to be more essential for maintaining the stability of the entire network. CytoHubba, a plugin in Cytoscape, was used to calculate the degree of each protein node. We considered the top 10 identified genes as the hub genes in our study.

\subsection{Identification of Hub Genes Affecting PD Progression.} GraphPad Prism 8, graphing software that can perform data analysis and data visualization, was used to analyze the expression of these 10 hub genes at different stages of PD. We downloaded the clinical data of GSE42966 from the GEO database and extracted the expression data for the hub genes in Braak 3 and Braak 4 PD stages. Next, we divided the patients with PD into two groups according to different stages (Braak 3 and Braak 4) and finally used GraphPad Prism 8 to perform statistical analysis and plot graphs for the two groups of data.

\section{Results}

3.1. Identification of DEGs. GSE42966 series was selected for the present study. GSE42966 contained nine PD samples and six normal samples. Based on the criteria of $P<0.05$ and $\log \mathrm{FC} \mid \geq 1.0$, a total of 773 DEGs were identified from GSE42966, including 339 upregulated genes and 434 downregulated genes. All DEGs were identified by comparing the $\mathrm{PD}$ samples with the normal samples of the substantia nigra. A volcano map of all identified DEGs is shown in Figure 1. All DEGs were arranged in the ascending order of the $P$ value since the smaller the $P$ value, the more significant the difference. A heat map of the top 50 genes is shown in Figure 2. Information on the top 20 genes is presented in Table 1 .

3.2. Functional Enrichment Analyses of DEGs. GO function and KEGG pathway enrichment analyses for DEGs were performed using DAVID (Figures 3 and 4). The enriched GO terms were divided into CC, BP, and MF ontologies. The results of the $\mathrm{GO}$ analysis indicated that DEGs were mainly enriched in MF, including protein binding, ATP binding, GTP binding, and protein kinase binding. CC analysis showed that DEGs were significantly enriched in the cytosol, extracellular exosomes, membranes, mitochondria, Golgi membranes, cell junctions, dendrites, neuronal cell bodies, centrosomes, postsynaptic membranes, microtubules, myelin sheaths, and axons. BP analysis showed that DEGs were significantly enriched in synaptic transmission and transport. In addition, the results of the KEGG pathway analysis showed that DEGs were mainly enriched in pathways involved in metabolism, Huntington's disease, biosynthesis of 


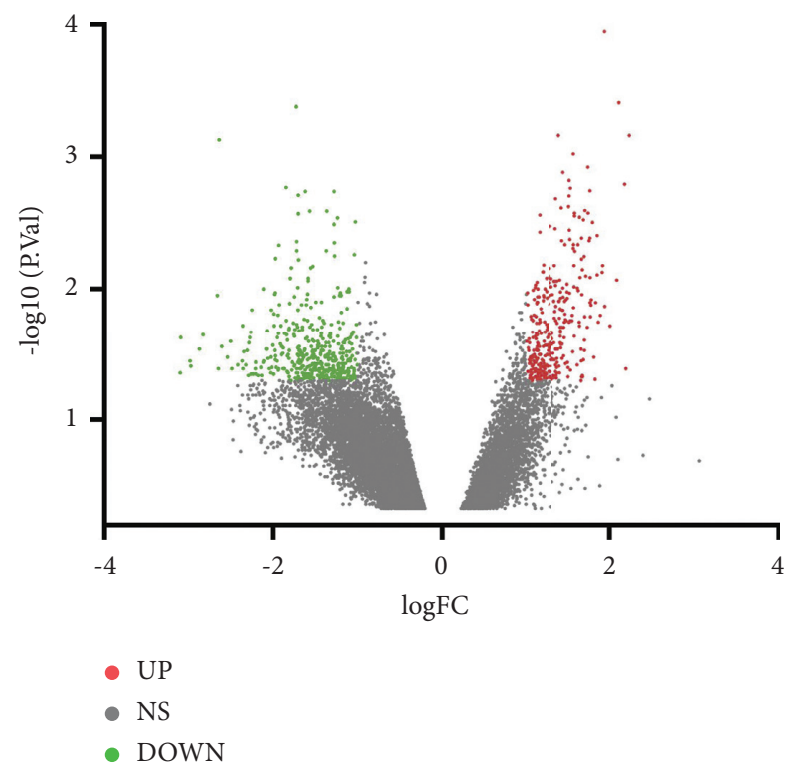

Figure 1: Volcano maps of all DEGs. Red represents upregulated genes, green represents downregulated genes, and gray represents genes that are not differentially expressed.

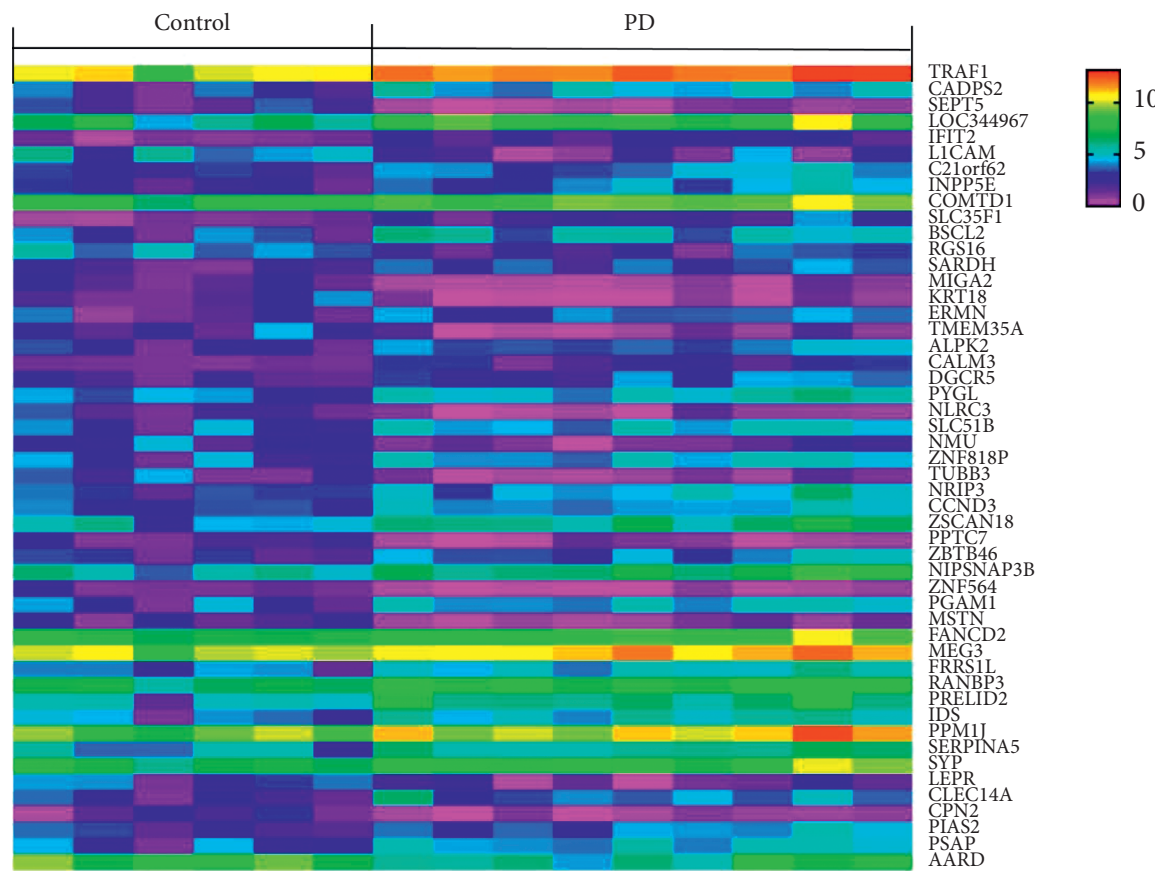

FIGURE 2: Heat map of the top 50 DEGs.

antibiotics, dopaminergic synapses, Alzheimer's disease, synaptic vesicle cycle, carbon metabolism, PD, GABAergic synapse, oxidative phosphorylation, oxytocin signaling pathway, phagosome, gap junction, circadian entrainment, retrograde endocannabinoid signaling, serotonergic synapse, cholinergic synapse, adrenergic signaling in cardiomyocytes, glucagon signaling pathway, aldosterone synthesis and secretion, estrogen signaling pathway, and melanogenesis.
3.3. PPI Network Construction and Hub Gene Identification. Protein interactions among the DEGs were predicted using the STRING tool. A total of 774 nodes were involved in the PPI network, as shown in Figure 5. The top 10 hub genes evaluated by the degree of connectivity in the PPI network were identified (Table 2 and Figure 6). The results showed that GNG2 was the most outstanding gene with connectivity degree $=27$, followed by GNG3 $($ degree $=24)$, PRKACA (degree $=23)$, HRAS $\quad($ degree $=23)$, MAPK1 
Table 1: The top 20 of DEGs.

\begin{tabular}{lccc}
\hline Gene & $P$ value & Log FC & Description \\
\hline TRAF1 & 0.000112 & 1.9436568 & Up \\
CADPS2 & 0.000392 & 2.112701 & Up \\
SEPT5 & 0.000417 & -1.7267324 & Down \\
LOC344967 & 0.000688 & 2.2360217 & Up \\
IFIT2 & 0.00069 & 1.3968911 & Up \\
L1CAM & 0.000747 & -2.6371341 & Down \\
C21orf62 & 0.000961 & 1.572589 & Up \\
INPP5E & 0.001201 & 1.7463712 & Up \\
COMTD1 & 0.001327 & 1.4499857 & Up \\
SLC35F1 & 0.001518 & 1.5228036 & Up \\
BSCL2 & 0.001626 & 2.1813648 & Up \\
RGS16 & 0.001695 & -1.8477099 & Down \\
SARDH & 0.00175 & 1.5392507 & Up \\
MIGA2 & 0.001801 & -1.2784036 & Down \\
KRT18 & 0.001817 & -1.6184519 & Down \\
ERMN & 0.001822 & 1.7714611 & Up \\
TMEM35A & 0.001953 & -1.703526 & Down \\
ALPK2 & 0.001989 & 1.5238576 & Up \\
CALM3 & 0.002103 & 1.3650642 & Up \\
DGCR5 & 0.002411 & 1.5177274 & Up \\
\hline P & &
\end{tabular}

$P$ value was used for describing the significance of the expression difference between the PD group and the healthy control group. The smaller the $p$ value was, the more significant the difference was.

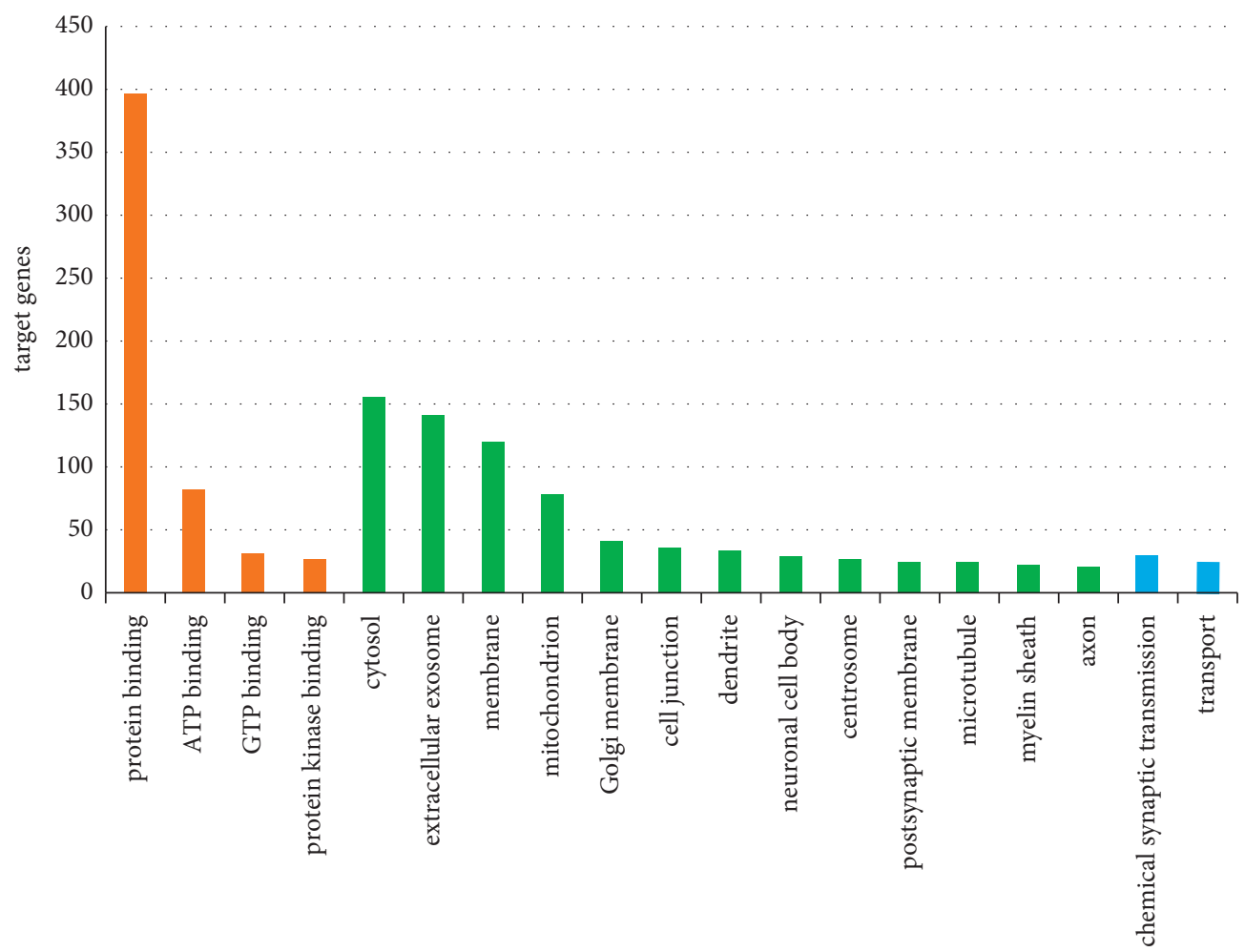

FIGURE 3: GO function enrichment analysis of DEGs. Orange represents MF, green represents CC, and blue represents BP. The numbers on the $y$-axis are gene counts.

$($ degree $=22), H S P A 8($ degree $=20)$, FPR1 $($ degree $=19)$, $A T P 5 B($ degree $=19), P S A P($ degree $=18)$, and GABBR2 (degree =18). Among them, GNG3, MAPK1, FPR1, and $A T P 5 B$ were downregulated in PD, and GNG2, PRKACA, HRAS, HSPA8, PSAP, and GABBR2 were upregulated in
PD. These 10 hub genes were significantly enriched in the six KEGG signaling pathways (Table 3), including the estrogen signaling pathway, serotonergic synapse, cholinergic synapse, chemokine signaling pathway, RAS signaling pathway, and pathways in cancer. 


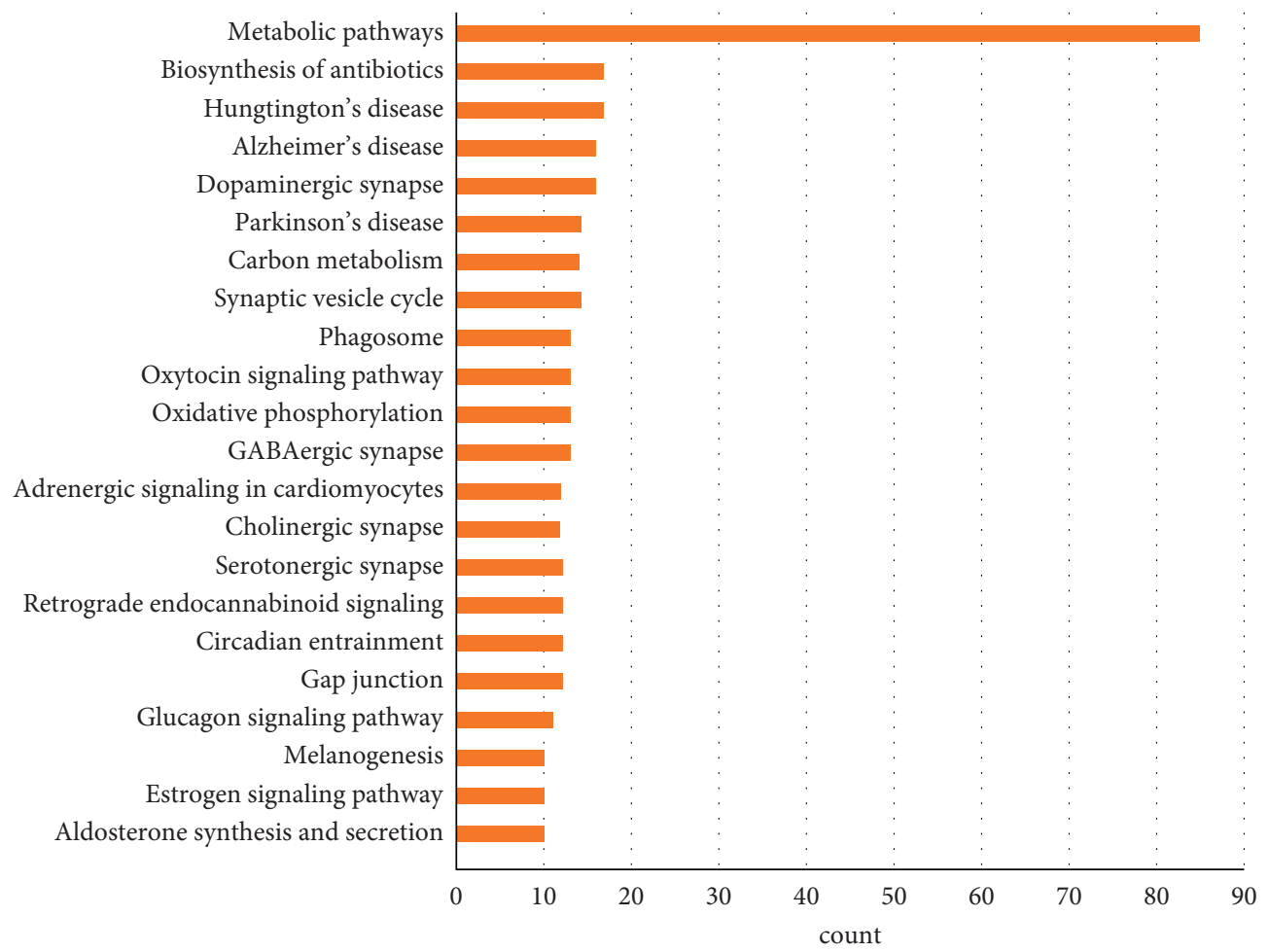

FIGURE 4: Enrichment analysis of the KEGG signaling pathway of DEGs. The numbers on the $x$-axis are gene counts.

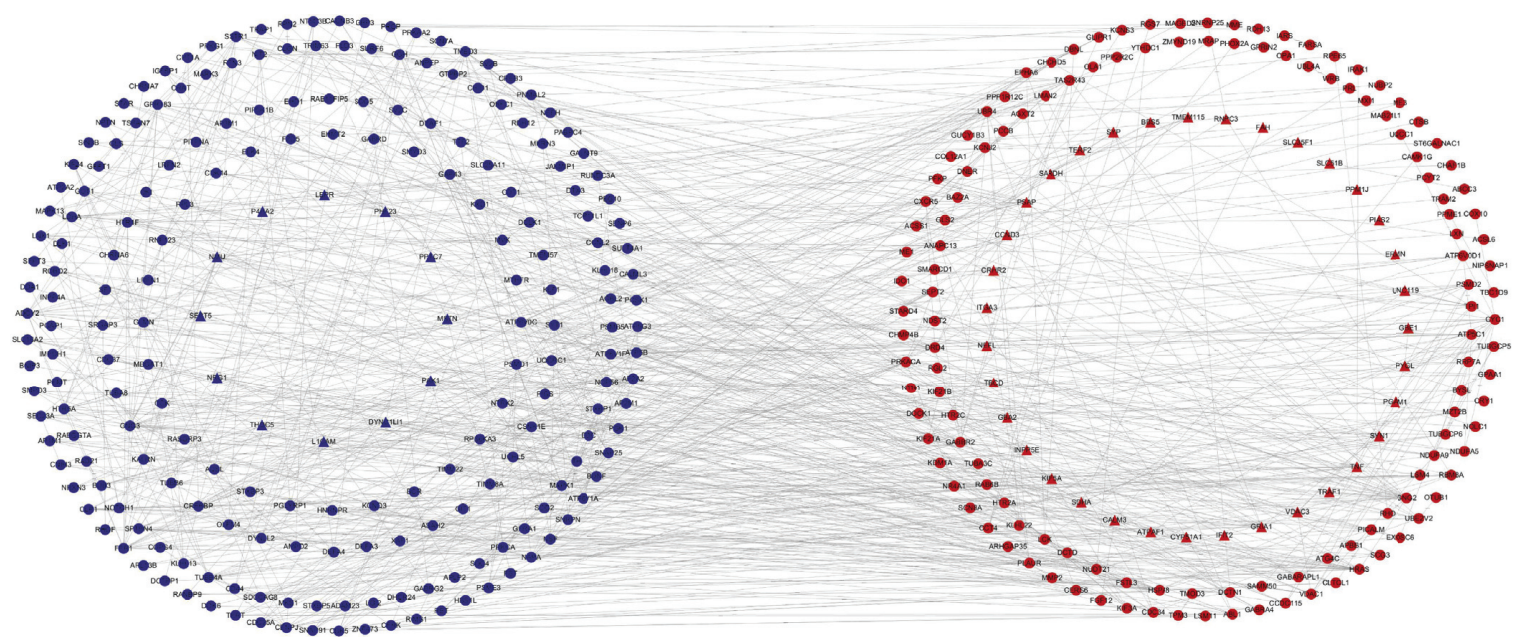

Figure 5: PPI network of DEGs. Blue represents downregulated genes, red represents upregulated genes, and the genes represented by triangles have more significant differences in gene expression than those represented by circles.

3.4. Identification of Hub Genes Affecting PD Progression. To investigate the values of the 10 hub genes in the progression of $\mathrm{PD}$, we downloaded the clinical data of GSE42966 from the GEO database and extracted the expression data of the hub genes in Braak 3 and Braak 4 stages. We then divided the patients with PD into two groups according to different Braak stages (Braak 3 and Braak 4). Among them, Braak 3 group contained four samples, and Braak 4 group contained five samples. Finally, we performed statistical analysis for the data from the two groups and found that the expression of the upregulated gene HRAS in PD was higher in Braak 4 than in Braak 3, the difference being statistically significant (Figure 7). There were no significant differences in the expression of the other nine hub genes in different stages.

\section{Discussion}

$\mathrm{PD}$ is the second most common age-related neurodegenerative disease [13]. Genetic factors are known to play an important role in the pathogenesis of PD [3]. Till date, at least 28 genes are known to be related to the pathogenesis of PD [14], but the identity of genes involved in $\mathrm{PD}$ progression remains unclear, especially key genes that 
TABLE 2: List of top 10 differentially expressed genes with higher degrees in the PPI network.

\begin{tabular}{lcc}
\hline Gene & Description & Degree \\
\hline GNG2 & $\mathrm{Up}$ & 27 \\
GNG3 & Down & 24 \\
PRKACA & $\mathrm{Up}$ & 23 \\
HRAS & $\mathrm{Up}$ & 23 \\
MAPK1 & Down & 22 \\
HSPA8 & $\mathrm{Up}$ & 20 \\
FPR1 & Down & 19 \\
ATP5B & Down & 19 \\
PSAP & $\mathrm{Up}$ & 18 \\
GABBR2 & $\mathrm{Up}$ & 18 \\
\hline
\end{tabular}

Degree was used for describing the importance of protein nodes in the network. The higher the degree was, the more important the nodes were in the network.

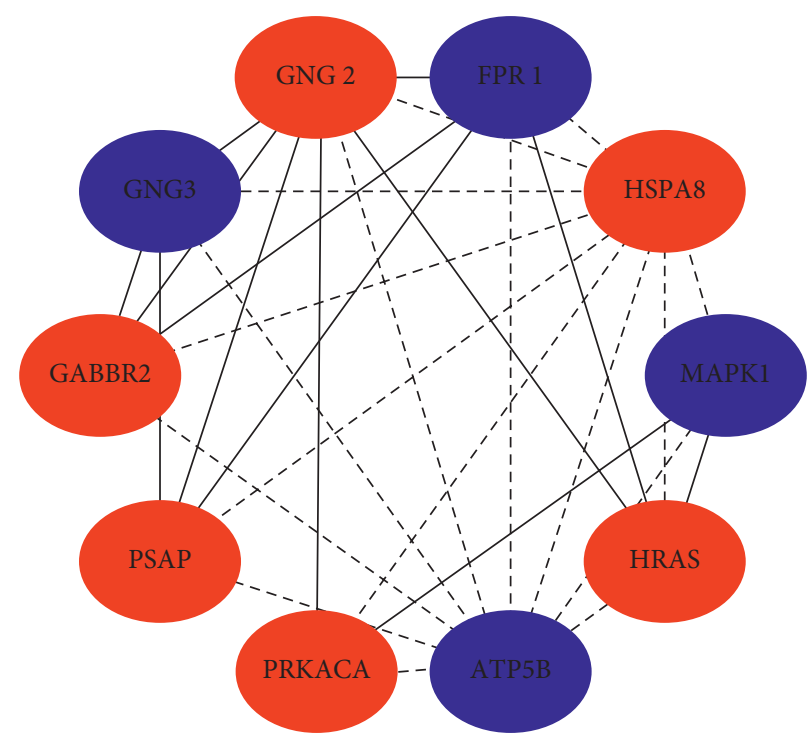

Figure 6: The top 10 hub genes in the PPI network. Blue represents downregulated genes, red represents upregulated genes, solid lines represent direct interactions between genes, and dotted lines represent indirect interactions between genes.

TABLE 3: List of KEGG pathways of hub genes.

\begin{tabular}{|c|c|c|c|c|}
\hline Pathway ID & Pathway name & Count & $P$ value & Genes \\
\hline hsa04915 & Estrogen signaling pathway & 5 & $4.81 E-06$ & PRKACA, HSPA8, GABBR2, MAPK1, HRAS \\
\hline hsa04726 & Serotonergic synapse & 5 & $7.60 E-06$ & PRKACA, MAPK1, GNG2, GNG3, HRAS \\
\hline hsa04725 & Cholinergic synapse & 5 & $7.60 E-06$ & PRKACA, MAPK1, GNG2, GNG3, HRAS \\
\hline hsa04062 & Chemokine signaling pathway & 5 & $5.86 E-05$ & PRKACA, MAPK1, GNG2, GNG3, HRAS \\
\hline hsa04014 & RAS signaling pathway & 5 & $1.26 E-04$ & PRKACA, MAPK1, GNG2, GNG3, HRAS \\
\hline hsa05200 & Pathways in cancer & 5 & 0.001050431 & PRKACA, MAPK1, GNG2, GNG3, HRAS \\
\hline
\end{tabular}

KEGG: Kyoto Encyclopedia of Genes and Genomes.

can predict disease progression. In this study, we identified a total of 733 DEGs that were significantly enriched in metabolic pathways. PPI network analysis found that 10 hub genes may be involved in the pathogenesis of PD. Among these, HRAS has the potential to predict the progression of PD.

The significant enrichment of DEGs in metabolic pathways as identified by this study suggests that this pathway may play an important role in the pathogenesis of PD. A previous study [15] demonstrated that multiple metabolic pathways are involved in PD. Tan et al. [16] also found the important value of metabolic pathways in the pathogenesis of PD by analyzing GEO dataset GSE72267, supporting the results from our study.

In this study, we also found that compared to the control group, the expression of HRAS was upregulated in patients with PD, with differences observed in expression between patients with PD with Braak 4 and Braak 3 stages, suggesting that HRAS expression may have the potential to predict the occurrence and progression of PD. HRAS is a member of the $R A S$ oncogene family, and its mutations are common in a variety of cancers, including head and neck cancer, skin cancer, and hematopoietic cancers [17, 18]. However, the relationship between $H R A S$ and $\mathrm{PD}$ has rarely been 


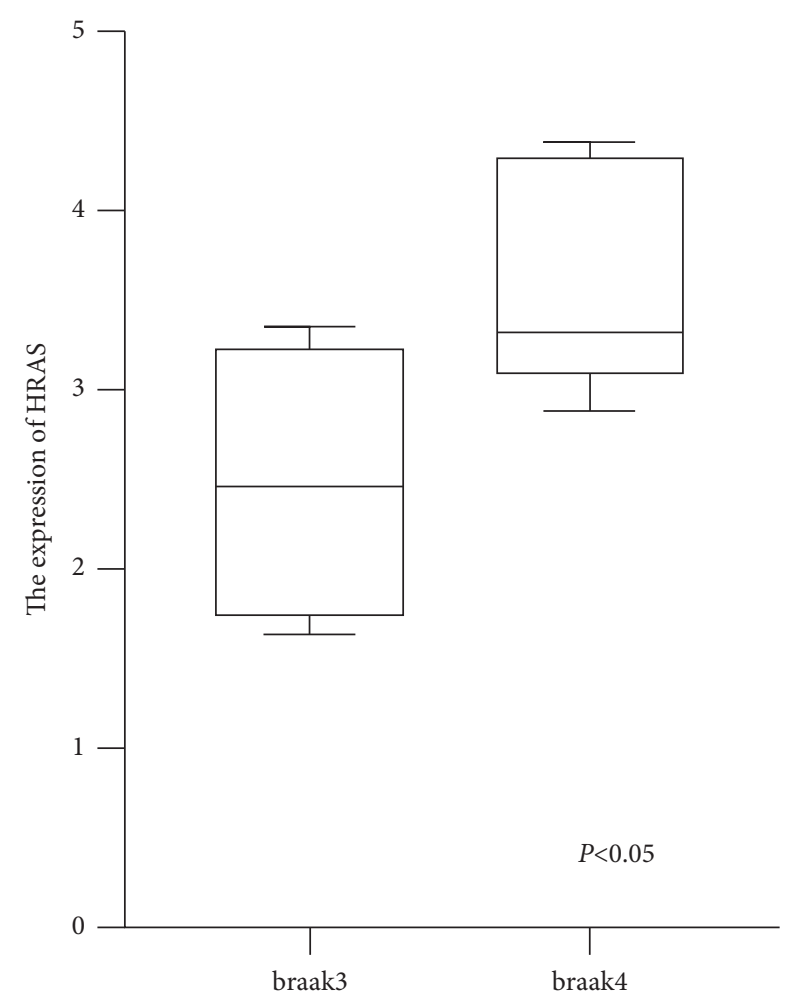

FIgURE 7: The expression of HRAS in brain tissues of patients with Braak 3 and Braak 4.

reported. Masuda et al. [19] found that the activation of the $\mathrm{H}$-RAS/ERK signaling pathway plays an important role in the NO/ROS redox signal-mediated cell model of PD, suggesting that HRAS is involved in the pathogenesis of PD. $H R A S$ is a GTPase protein that can activate the mTOR signaling pathway [20]. mTOR plays a key role in brain development, neuronal survival, synaptic plasticity, and memory formation [21] and is related to the pathogenesis of neurodegenerative diseases, especially PD [22]. mTOR is a serine/threonine kinase that is the core component of the mTORC1 and mTORC2 multiprotein complex. mTORC1 controls protein translation and autophagy [23]. Autophagy is the main degradation pathway that eliminates various aggregate proteins, and autophagy activation defects are common in many neurodegenerative diseases, such as PD [24]. Therefore, we speculate that HRAS overexpression may inhibit autophagy and promote the occurrence and development of PD by activating the mTOR pathway. In addition, results from our research showed that HRAS genes were significantly enriched in the following KEGG signaling pathways: estrogen signaling pathway, serotonergic synapse, cholinergic synapse, chemokine signaling pathway, RAS signaling pathway, and pathways in cancer. Among them, cholinergic synaptic signaling pathway dysfunction has been confirmed to be related to cognitive impairment in patients with PD [25], suggesting that HRAS may be involved in the pathogenesis of PD dementia; the specific mechanism remains to be confirmed by further studies.

Another study [26] showed that HRAS rs12628 is related to L-DOPA-induced dyskinesia (LID). LID is one of the most ineffective adverse reactions in the treatment of PD. Approximately $80 \%$ of patients with $\mathrm{PD}$ who receive levodopa treatment will develop LID within 5-10 years after starting DA replacement therapy [27]. This suggests that indepth research on HRAS is also particularly important for the treatment of PD and may help prevent early and/or severe LID. HRAS could be potentially used as a target for the treatment of PD.

Prosaposin, encoded by the PSAP gene, is a multifunctional protein containing four saposin domains A-D, with neuroprotective, neurotrophic, and lysosomal function regulation effects [28]. Previous studies have shown that PSAP exerts a neuroprotective effect in PD models [29], and saposin deficiency can lead to age-dependent neurodegeneration [30]. Oji et al. [31] identified two PSAP saposin D-domain intron variants, rs4747203 and rs885828, in patients with autosomal dominant PD from Japan and Taiwan, which are related to susceptibility to PD. They also found that PSAP saposin D mutations can cause progressive motor decline and dopaminergic neurodegeneration in mice. Lin et al. [32] also found that the frequency of the rs142614739 variant of the PSAP saposin D domain in PD increased in the Eastern Chinese population, which is a risk factor for PD. Our research shows that the expression of the PSAP gene is upregulated in patients with PD and may be involved in the pathogenesis of $\mathrm{PD}$, which is consistent with the above research results.

Our study showed that protein kinase A catalytic subunit (PRKACA) is highly expressed in the substantia nigra of patients with $\mathrm{PD}$, and the KEGG enrichment pathway shows that it is involved in the dopamine and acetylcholinergic pathways. Chi et al. [33] analyzed DEGs in the GSE6613 dataset in the GEO database and found that PRKACA was upregulated in patients with $\mathrm{PD}$ and may be related to PD. Jia et al. [34] analyzed DEGs in different brain regions of the PD mouse model and found that PRKACA is one of the proteins in the PPI network that interacts closely with other proteins and may participate in the pathogenesis of PD by causing an imbalance in DA and acetylcholine. These conclusions are consistent with the results of this study.

$M A P K 1$ is a serine-threonine kinase belonging to the MAPK family. Excessive activation of MAPK can cause neuronal death in PD [35]. Previous studies have shown that $M A P K 1$ is associated with the progression of $\mathrm{PD}$ [36]. The accumulation of phosphorylated MAPK in the halo area of Lewy bodies also supports its pathogenic role in PD [37]. In addition, fibroblasts from patients with PD with the G2019S LRRK2 mutation showed excessive activation of the MAPK1/3 pathway [38], and knockdown of long noncoding RNA SNHG1 can improve apoptosis, oxidative stress, and inflammation in PD models by inhibiting the miR-125b-5p/ MAPK1 axis [39], which indicates that MAPK1 is involved in the pathogenesis of PD. Our results show that, compared to the control group, MAPK1 is abnormally expressed in PD and is the hub gene in PPI, suggesting its important role in the pathogenesis of $\mathrm{PD}$, consistent with the aforementioned research results.

$\mathrm{PD}$ is well known to be caused by the progressive death of DA neurons in the substantia nigra of the midbrain, 
leading to a lack of DA in the striatum. Studies have shown that chronic DA depletion can enhance the transmission of GABA in the striatum, thus affecting cell and circuit activities, which are related to the pathogenesis of PD [40]. A study has shown [41] that patients with PD in Braak 3 exhibit an increased threshold of GABA medium spiny neurons, and patients with PD in Braak 4 exhibit decreased spontaneous GABA activity. The "GABA collapse" hypothesis proposed by Błaszczyk suggests that GABA imbalance plays an important role in the development and progression of $\mathrm{PD}$ and other neurodegenerative diseases. GABBR2 is the main gene involved in GABA signal transduction and plays a vital role in maintaining the balance of excitation/inhibition in brain synapses [42]. Other studies have shown that GNG3 may be related to the GABAergic synaptic pathway [43], and its destruction may lead to dysfunction of the GABAB1 receptor signaling pathway [44]. Our results suggest that GABBR2 and GNG3 are hub genes in the PPI network, and KEGG pathway enrichment analysis showed that they were significantly enriched in the GABAergic synaptic pathway, suggesting that they may participate in the pathogenesis of PD by affecting GABAergic synapse signaling.

Studies have shown that mitochondrial dysfunction is an important pathogenic mechanism that leads to motor dysfunction and damage to dopaminergic neurons in patients with PD $[45,46] . A T P 5 B$ is an important part of the mitochondrial respiratory chain complex and catalyzes the synthesis of ATP [47]. Our results show that the expression of $A T P 5 B$ is downregulated in PD. It is speculated that PD may occur by affecting mitochondrial function, but the specific mechanism needs to be confirmed by further studies.

In addition, our research also showed that GNG2 and HSPA8 were upregulated in PD and were the hub genes in PPI. Lyu et al. [48] also found that GNG2 may play a key role in the pathogenesis of PD by analyzing gene expression in different brain regions in a PD mouse model. Molochnikov et al. [49] found that HSPA8 is abnormally expressed in blood of patients with PD compared to healthy individuals and has the potential to predict PD. These studies support our findings. Our study also shows that FPR1 is downregulated in the substantia nigra of patients with PD and plays an important role as a hub gene. Recent studies have shown that FPR1 activation can promote the proliferation and differentiation of nerve cells by activating reactive oxygen species and plays an important role in neurological diseases [50,51]. Therefore, we speculated that it may participate in the occurrence of PD by inhibiting neuronal proliferation and differentiation. However, little is known about the role of these genes in PD, and further research is needed in the future.

This study has some shortcomings, including that the study only contains one series, and the number of samples is small. Further studies are warranted to confirm the results of this study. Therefore, we are going to establish in vitro and in vivo experimental models to verify our conclusions.

\section{Conclusion}

In conclusion, our data demonstrated that metabolic pathways play an important role in the pathogenesis of PD. Ten hub genes (GNG3, MAPK1, FPR1, ATP5B, GNG2, PRKACA, HRAS, HSPA8, PSAP, and GABBR2) may be involved in the pathogenesis of PD, among which HRAS may have potential value in predicting the progression of PD. However, due to the aforementioned limitations of the study, further investigation is needed in the future.

\section{Abbreviations}

PD: $\quad$ Parkinson's disease

DEGs: Differentially expressed genes

PPI: $\quad$ Protein-protein interaction

GNG3: $\quad$ G protein subunit gamma 3

MAPK1: Mitogen-activated protein kinase 1

FPR1: $\quad$ Formyl peptide receptor 1

ATP5B: $\quad$ ATP synthase F1 subunit beta

GNG2: $\quad$ G protein subunit gamma 2

PRKACA: Protein kinase A catalytic subunit

HRAS: $\quad$ HRas proto-oncogene, GTPase

HSPA8: $\quad$ Heat shock $70 \mathrm{kDa}$ protein 8

PSAP: $\quad$ Prosaposin

GABBR2: Gamma-aminobutyric acid type B receptor subunit 2

LRRK2: $\quad$ Leucine-rich repeat kinase 2

VPS35: VPS35 retromer complex component

GEO: $\quad$ Gene Expression Omnibus

GO: Gene Ontology

KEGG: $\quad$ Kyoto Encyclopedia of Genes and Genomes

BP: Biological process

MF: $\quad$ Molecular function

CC: $\quad$ Cellular component

DAVID: Database for Annotation, Visualization, and Integrated Discovery

STRING: $\quad$ Search Tool for Recurring Instances of Neighbouring Genes

TRAF1: $\quad$ TNF receptor-associated factor 1

CADPS2: Calcium-dependent secretion activator 2

SEPT5: $\quad$ Septin 5

LOC344967: Acyl-CoA thioesterase 7 pseudogene

IFIT2: Interferon-induced protein with tetratricopeptide repeats 2

L1CAM: $\quad$ L1 cell adhesion molecule

C21orf62: Chromosome 21 open reading frame 62

INPP5E: Inositol polyphosphate-5-phosphatase E

COMTD1: Catechol-O-methyltransferase domaincontaining 1

SLC35F1: Solute carrier family 35 member F1

BSCL2: $\quad$ Berardinelli-Seip congenital lipodystrophy type 2 protein

RGS16: Regulator of G-protein signaling 16

SARDH: Sarcosine dehydrogenase

MIGA2: Mitoguardin 2

KRT18: Keratin 18

ERMN: Ermin

TMEM35A: Transmembrane protein 35A 


$\begin{array}{ll}\text { ALPK2: } & \text { Alpha kinase } 2 \\ \text { CALM3: } & \text { Calmodulin } 3 \\ \text { DGCR5: } & \text { DiGeorge syndrome critical region gene } 5 \\ \text { ATP: } & \text { Adenosine triphosphate } \\ \text { GTP: } & \text { Guanosine triphosphate } \\ \text { GMP: } & \text { Guanosine monophosphate } \\ \text { GABA: } & \gamma \text {-Aminobutyrate } \\ \text { mTORC: } & \text { Mechanistic target of rapamycin complex } \\ \text { L-DOPA: } & \text { Levodopa } \\ \text { LID: } & \text { L-DOPA-induced dyskinesia } \\ \text { NO: } & \text { Nitric oxide } \\ \text { ROS: } & \text { Reactive oxygen species } \\ \text { DA: } & \text { Dopamine. }\end{array}$

\section{Data Availability}

In this study, gene expression datasets were obtained from the GEO database (https://www.ncbi.nlm.nih.gov/geo/). After careful review, we chose the series GSE42966 with clinical information on Braak staging. GSE42966 was based on the Agilent GPL4133 platform (Agilent-014850 Whole Human Genome Microarray 4x44K G4112F). The data are freely available online, and our study did not involve any experiments with humans or animals performed by any of the authors.

\section{Conflicts of Interest}

The authors declare that there are no conflicts of interest regarding the publication of this article.

\section{Acknowledgments}

This study was supported by the Jilin Province Center for Precision Medicine Diagnosis and Treatment of Nervous System Diseases (no. 20200602045ZP), Department of Science and Technology of Jilin Province, and Research on the Mechanism of HNRNP's Participation in Parkinson's Disease, Health Special Project, Department of Finance of Jilin Province. The authors thank the Department of Finance of Jilin Province and the Department of Science and Technology of Jilin Province for the support.

\section{References}

[1] B. L. B. Marino, L. R. de Souza, K. P. A. Sousa et al., "Parkinson's disease: a review from pathophysiology to treatment," Mini Reviews in Medicinal Chemistry, vol. 20, no. 9, pp. 754-767, 2020.

[2] A. H. V. Schapira, "Neurobiology and treatment of Parkinson's disease," Trends in Pharmacological Sciences, vol. 30, no. 1, pp. 41-47, 2009.

[3] J. Gao, M. A. Nalls, M. Shi et al., "An exploratory analysis on gene-environment interactions for Parkinson disease," Neurobiology of Aging, vol. 33, no. 10, pp. 2528-2536, 2012.

[4] E. Tolosa, M. Vila, C. Klein, and O. Rascol, "LRRK2 in Parkinson disease: challenges of clinical trials," Nature Reviews Neurology, vol. 16, no. 2, pp. 97-107, 2020.

[5] A. Zimprich, A. Benet-Pagès, W. Struhal et al., "A mutation in VPS35, encoding a subunit of the retromer complex, causes late-onset Parkinson disease," The American Journal of $\mathrm{Hu}$ man Genetics, vol. 89, no. 1, pp. 168-175, 2011.

[6] H. Braak, U. Rüb, W. P. Gai, and K. Del Tredici, "Idiopathic Parkinson's disease: possible routes by which vulnerable neuronal types may be subject to neuroinvasion by an unknown pathogen," Journal of Neural Transmission, vol. 110, no. 5, pp. 517-536, 2003.

[7] H. Braak, K. D. Tredici, U. Rüb, R. A. I. de Vos, E. N. H. Jansen Steur, and E. Braak, "Staging of brain pathology related to sporadic Parkinson's disease," Neurobiology of Aging, vol. 24, no. 2, pp. 197-211, 2003.

[8] I. Hulsegge, A. Kommadath, and M. A. Smits, "Globaltest and GOEAST: two different approaches for gene ontology analysis," BMC Proceedings, vol. 3, no. Suppl 4, p. S10, 2009.

[9] D. Huang, B. T. Sherman, Q. Tan et al., "The DAVID gene functional classification tool: a novel biological modulecentric algorithm to functionally analyze large gene lists," Genome Biology, vol. 8, no. 9, p. R183, 2007.

[10] D. Szklarczyk, A. L. Gable, D. Lyon et al., "STRING v11: protein-protein association networks with increased coverage, supporting functional discovery in genome-wide experimental datasets," Nucleic Acids Research, vol. 47, no. D1, pp. D607-d613, 2019.

[11] D. Szklarczyk, J. H. Morris, H. Cook et al., "The string database in 2017: quality-controlled protein-protein association networks, made broadly accessible," Nucleic Acids Research, vol. 45, no. D1, pp. D362-d368, 2017.

[12] R. Saito, M. E. Smoot, K. Ono et al., "A travel guide to cytoscape plugins," Nature Methods, vol. 9, no. 11, pp. 1069-1076, 2012.

[13] M. S. Massaquoi, W. A. Liguore, M. J. Churchill, C. Moore, H. L. Melrose, and C. K. Meshul, "Gait deficits and loss of striatal tyrosine hydroxlase/trk-B are restored following 7,8dihydroxyflavone treatment in a progressive MPTP mouse model of Parkinson's disease," Neuroscience, vol. 433, pp. 53-71, 2020.

[14] M. A. Nalls, N. Pankratz, C. M. Lill et al., "Large-scale metaanalysis of genome-wide association data identifies six new risk loci for Parkinson's disease," Nature Genetics, vol. 46, no. 9, pp. 989-993, 2014.

[15] M. Bonin, S. Poths, H. Osaka, Y.-L. Wang, K. Wada, and O. Riess, "Microarray expression analysis of gad mice implicates involvement of Parkinson's disease associated UCHL1 in multiple metabolic pathways," Molecular Brain Research, vol. 126, no. 1, pp. 88-97, 2004.

[16] C. Tan, X. Liu, and J. Chen, "Microarray analysis of the molecular mechanism involved in Parkinson's disease," Parkinson's Disease, vol. 2018, Article ID 1590465, 12 pages, 2018.

[17] E. Sheffels and R. L. Kortum, "The role of wild-type RAS in oncogenic RAS transformation," Genes, vol. 12, no. 5, 2021.

[18] Y. Pylayeva-Gupta, E. Grabocka, and D. Bar-Sagi, "RAS oncogenes: weaving a tumorigenic web," Nature Reviews Cancer, vol. 11, no. 11, pp. 761-774, 2011.

[19] K. Masuda, H. Tsutsuki, S. Kasamatsu et al., "Involvement of nitric oxide/reactive oxygen species signaling via 8-nitrocGMP formation in 1-methyl-4-phenylpyridinium ion-induced neurotoxicity in PC12 cells and rat cerebellar granule neurons," Biochemical and Biophysical Research Communications, vol. 495, no. 3, pp. 2165-2170, 2018.

[20] M. E. Pacold, S. Suire, O. Perisic et al., "Crystal structure and functional analysis of ras binding to its effector phosphoinositide 3-kinase $\gamma$," Cell, vol. 103, no. 6, pp. 931-944, 2000. 
[21] J. Bockaert and P. Marin, "mTOR in brain physiology and pathologies," Physiological Reviews, vol. 95, no. 4, pp. 1157-1187, 2015.

[22] L. S. Tain, H. Mortiboys, R. N. Tao, E. Ziviani, O. Bandmann, and A. J. Whitworth, "Rapamycin activation of 4E-BP prevents parkinsonian dopaminergic neuron loss," Nature Neuroscience, vol. 12, no. 9, pp. 1129-1135, 2009.

[23] S. Sarkar, B. Ravikumar, R. A. Floto, and D. C. Rubinsztein, "Rapamycin and mTOR-independent autophagy inducers ameliorate toxicity of polyglutamine-expanded huntingtin and related proteinopathies," Cell Death \& Differentiation, vol. 16, no. 1, pp. 46-56, 2009.

[24] H. Park, J. H. Kang, and S. Lee, "Autophagy in neurodegenerative diseases: a hunter for aggregates," International Journal of Molecular Sciences, vol. 21, no. 9, 2020.

[25] N. S. Narayanan, R. L. Rodnitzky, and E. Y. Uc, "Prefrontal dopamine signaling and cognitive symptoms of Parkinson's disease," Reviews in the Neurosciences, vol. 24, no. 3, pp. 267-278, 2013.

[26] N. Martín-Flores, R. Fernández-Santiago, F. Antonelli et al., "MTOR pathway-based discovery of genetic susceptibility to L-DOPA-induced dyskinesia in Parkinson's disease patients," Molecular Neurobiology, vol. 56, no. 3, pp. 2092-2100, 2019.

[27] M. F. Bastide, W. G. Meissner, B. Picconi et al., "Pathophysiology of L-dopa-induced motor and non-motor complications in Parkinson's disease," Progress in Neurobiology, vol. 132, pp. 96-168, 2015.

[28] Y.-P. Chen, X.-J. Gu, R.-W. Ou et al., "Genetic analysis of prosaposin, the lysosomal storage disorder gene in Parkinson's disease," Molecular Neurobiology, vol. 58, no. 4, pp. 1583-1592, 2021.

[29] A. M. Nicholson, N. A. Finch, M. Almeida et al., "Prosaposin is a regulator of progranulin levels and oligomerization," Nature Communications, vol. 7, no. 1, p. 11992, 2016.

[30] S. J. Hindle, S. Hebbar, D. Schwudke, C. J. H. Elliott, and S. T. Sweeney, "A saposin deficiency model in drosophila: lysosomal storage, progressive neurodegeneration and sensory physiological decline," Neurobiology of Disease, vol. 98, pp. 77-87, 2017.

[31] Y. Oji, T. Hatano, S.-I. Ueno et al., "Variants in saposin D domain of prosaposin gene linked to Parkinson's disease," Brain, vol. 143, no. 4, pp. 1190-1205, 2020.

[32] Z.-H. Lin, Y. Ruan, N.-J. Xue, Y. Fang, J.-L. Pu, and B.-R. Zhang, "PSAP intronic variants around saposin D domain and Parkinson's disease," Brain, vol. 144, no. 1, p. e3, 2021.

[33] L. M. Chi, L. P. Wang, and D. Jiao, "Identification of differentially expressed genes and long noncoding RNAs associated with Parkinson's disease," Parkinson's Disease, vol. 2019, Article ID 6078251, 7 pages, 2019.

[34] E. Jia, M. Pan, Z. Liu et al., "Transcriptomic profiling of differentially expressed genes and related pathways in different brain regions in Parkinson's disease," Neuroscience Letters, vol. 732, Article ID 135074, 2020.

[35] E. K. Kim and E.-J. Choi, "Pathological roles of MAPK signaling pathways in human diseases," Biochimica et Biophysica Acta-Molecular Basis of Disease, vol. 1802, no. 4, pp. 396-405, 2010.

[36] J. C. Triplett, Z. Zhang, R. Sultana et al., "Quantitative expression proteomics and phosphoproteomics profile of brain from PINK1 knockout mice: insights into mechanisms of familial Parkinson's disease," Journal of Neurochemistry, vol. 133, no. 5, pp. 750-765, 2015.
[37] J.-H. Zhu, S. M. Kulich, T. D. Oury, and C. T. Chu, "Cytoplasmic aggregates of phosphorylated extracellular signalregulated protein kinases in lewy body diseases," American Journal Of Pathology, vol. 161, no. 6, pp. 2087-2098, 2002.

[38] J. M. Bravo-San Pedro, R. Gómez-Sánchez, M. Niso-Santano et al., "The MAPK1/3 pathway is essential for the deregulation of autophagy observed in G2019S LRRK2 mutant fibroblasts," Autophagy, vol. 8, no. 10, pp. 1537-1539, 2012.

[39] X. Xiao, Z. Tan, M. Jia et al., "Long noncoding RNA SNHG1 knockdown ameliorates apoptosis, oxidative stress and inflammation in models of Parkinson's disease by inhibiting the miR-125b-5p/MAPK1 Axis," Neuropsychiatric Disease and Treatment, vol. 17, pp. 1153-1163, 2021.

[40] A. Faynveitz, H. Lavian, A. Jacob, and A. Korngreen, "Proliferation of inhibitory input to the substantia nigra in experimental parkinsonism," Frontiers in Cellular Neuroscience, vol. 13, p. 417, 2019.

[41] J. W. Błaszczyk, "Parkinson's disease and neurodegeneration: GABA-collapse hypothesis," Frontiers in Neuroscience, vol. 10, p. 269, 2016.

[42] M. M. Pallotta, R. Ronca, R. Carotenuto et al., "Specific effects of chronic dietary exposure to chlorpyrifos on brain gene expression-A mouse study," International Journal of Molecular Sciences, vol. 18, no. 11, 2017.

[43] X. Chen, Y. Pan, M. Yan, G. Bao, and X. Sun, "Identification of potential crucial genes and molecular mechanisms in glioblastoma multiforme by bioinformatics analysis," Molecular Medicine Reports, vol. 22, no. 2, pp. 859-869, 2020.

[44] W. F. Schwindinger, K. E. Giger, K. S. Betz et al., "Mice with deficiency of G protein $\gamma 3$ are lean and have seizures," Molecular and Cellular Biology, vol. 24, no. 17, pp. 7758-7768, 2004.

[45] A. Bose and M. F. Beal, "Mitochondrial dysfunction in Parkinson's disease," Journal of Neurochemistry, vol. 139, no. Suppl 1, pp. 216-231, 2016.

[46] E. M. Rocha, B. De Miranda, and L. H. Sanders, "Alphasynuclein: pathology, mitochondrial dysfunction and neuroinflammation in Parkinson's disease," Neurobiology of Disease, vol. 109, no. Pt B, pp. 249-257, 2018.

[47] H. Wu, X. Liu, Z. Y. Gao, M. Lin, and X. Zhao, "Icaritin provides neuroprotection in Parkinson's disease by attenuating neuroinflammation, oxidative stress, and energy deficiency," Antioxidants, vol. 10, no. 4, 2021.

[48] Y. Lyu, Y. Huang, G. Shi, X. Lei, and K. Li, “Transcriptome profiling of five brain regions in a 6-hydroxydopamine rat model of Parkinson's disease," CNS Neuroscience \& Therapeutics, vol. 4, 2021.

[49] L. Molochnikov, J. M. Rabey, E. Dobronevsky et al., “A molecular signature in blood identifies early Parkinson's disease," Molecular Neurodegeneration, vol. 7, no. 1, p. 26, 2012.

[50] G. Wang, L. Zhang, X. Chen et al., "Formylpeptide receptors promote the migration and differentiation of rat neural stem cells," Scientific Reports, vol. 6, no. 1, p. 25946, 2016.

[51] L. Zhang, G. Wang, X. Chen et al., "Formyl peptide receptors promotes neural differentiation in mouse neural stem cells by ROS generation and regulation of PI3K-AKT signaling," Scientific Reports, vol. 7, no. 1, p. 206, 2017. 